\title{
Dynamic Analysis on Water Inrush from Fault in Floor ZHOU Zhihua ${ }^{1,2, a}$, CAO Ping ${ }^{1 . b}$, YE Zhouyuan ${ }^{2, c}$, WANG Yi-xian ${ }^{3, d}$
}

${ }^{1}$ School of Resources and Safety Engineering, Central South University, Changsha 410083, China

${ }^{2}$ School of Energy and Safety Engineering, Hunan University of Science and Technology, Xiangtan 411201, China

${ }^{3}$ School of Civil Engineering, Hefei University of Technology, Hefei, 230009, China

azhouzhihua17@126.com, ${ }^{\mathrm{b} p c a o \_c s u @ s i n a . c o m, ~}{ }^{\mathrm{c} y e z y}$ 7@126.com, ${ }^{\mathrm{d}}$ wangyixian2010@gmail.com

Keywords: fault; water inrush; dynamic analysis; stress intensity factor

\begin{abstract}
In this paper, the deficiency on present mechanism of water inrush from floor is analyzed and it is found that water inrush from fault not only relative with static loads, but also with dynamic loads, especially faults in deep are easily acted by low dynamic loads. Based on fracture mechanics, the fault activation model is built and the stress intensity factor is calculated in view of the deficiency on present mechanism of water inrush from floor. The influence on fault depth, fault length, fault span, fault thickness, underground water pressure is considered in the model for faults in deep. Whether the fault is active is judged by strain energy density factor. The strain energy density factor is relative with fault parameters. It is reverse with fault thickness, positively increases with fault depth and stope span. It also positively increases with dynamic loads and acting time.
\end{abstract}

\section{Introduction}

There are abundant coal resources in china, but most coal mines are seriously threatened by water disaster. With the increase of mining depth, the underground water pressure increases, flood threat is more and more serious. Water bursting from floor is the major water disaster facing coal mines. From the present mine water inrush statistics, most water inrush accidents occurred in the coal working face, and $80 \%$ of the water inrush accidents are due to the presence of faults, other water inrush accidents may be also due to the presence of buried fault ${ }^{[1]}$. Therefore, the problem of water inrush from coal floor is in fact the matter of water bursting from fault. To solve this problem, we should take the fault as main research object.

A lot of scholars have done many researches on water inrush through fault. Tan zhixiang ${ }^{[2]}$ analyzed the mechanics of water inrush from coal floor and from fault based on the limit equilibrium theory of rock mass. Yang xin'an ${ }^{[3]}$ classified water inrush by the initial characteristics

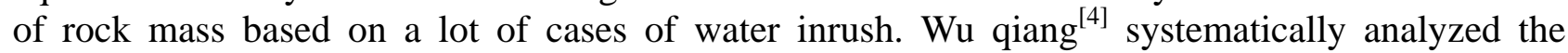
deformation and destruction patterns of fault materials under different moisture content, confining pressure and loading modes through compression-creep tests. Yin Huiyong ${ }^{[5]}$ and Zhang Jincai ${ }^{[6]}$ divide the water inrush from fault into two categories: water inrush from the water conductive fault which is formed by the transfixion of tectonic rock zones and floor crack, the other water inrush caused by fault activation because of mining. Li jianmin ${ }^{[7,8]}$ calculated the stress around rock at the different mining stages and analyzed the patterns and kinds of water inrush.

Many scholars believe that the stress field change caused the fault and fissure activation, expansion, mutual transfixion to format water channel, which is verified by many examples. But for the change of stress field are mainly considered from static or quasi-static loads, few from dynamic loads. In fact, the rock responses differ greatly from dynamic loads and static loads, fault water inrush mechanism should also be particularly considering dynamic loads disturbance. Especially for water inrush from fault in deep stope, due to the deep rock mass itself in high geo-stress field, in a state of limit equilibrium, it is vulnerable to external force effects and changes of state. The mechanism of water inrush from rock mass with a fault in the ground stress is analyzed from the angle of dynamic loads in this paper. 


\section{Mechanization of water gushing from fault structure}

According to the position of fault and tunnel floor, the fault is divided into semi-open fault, hidden fault and breakthrough fault as shown in Fig.1. Hidden fault doesn't link up with the tunnel floor, bur it will develop to the semi-open fault with the stress change when backstopping or tunneling. Hidden fault and semi-open fault will develop to the breakthrough one when the stress changes. So the three types of fault propagation mechanism study can be down to semi-open fault propagation mechanism research.

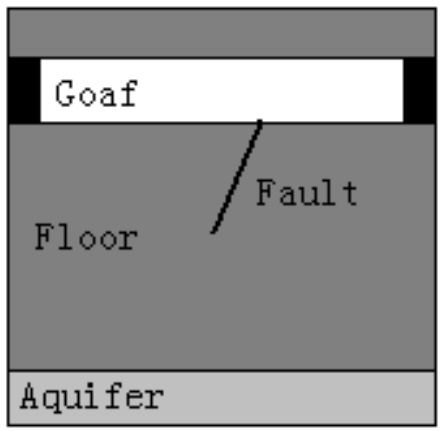

(a) semi-open fault

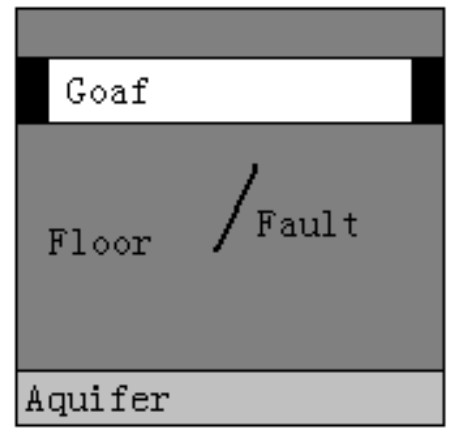

(b) hidden fault

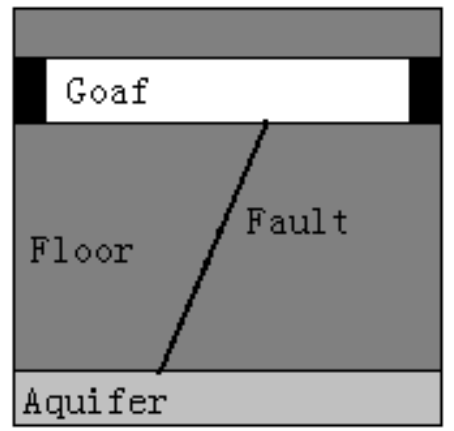

(c) breakthrough fault

Fig.1 Fault's distribution condition

In deep stope there is a semi-open fault, as shown in Figure 1 ( a ), the stress environment as shown in Figure 2. The fault length is a, dip angle is $\alpha$, the floor thickness is $\mathrm{W}$, the upper surface span of the floor is L. The upper floor rock is a stope or roadway, which is thought to be a free surface without force. The left and right of floor is around by the action of horizontal stress $\mathrm{P}_{\mathrm{s}}$, the lower is subjected to the vertical stress $\mathrm{P}_{\mathrm{v}}$ and water pressure $\mathrm{P}_{\mathrm{w}}$, the floor is also subjected to dynamic load $\mathrm{P}_{\mathrm{d}}$ with the horizontal direction at a $\gamma$ angle, as shown in Fig.2. The floor rock is regarded as homogeneous body. When water inrush from the floor rock, the fault must be first activated, develop and format to breakthrough one which provide channel for water inrush.

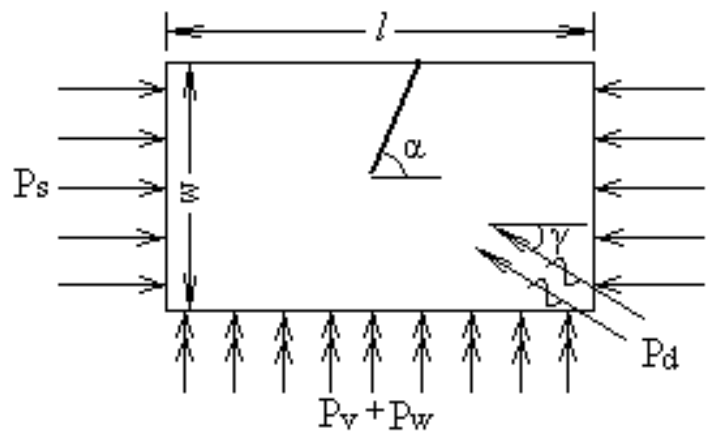

Fig.2 loading conditions of semi-open fault

According to the superposition principle, the stress in Fig.2 can be decomposed into three part loads in Fig.3, and the end Stress Intensity Factors are as fellows.

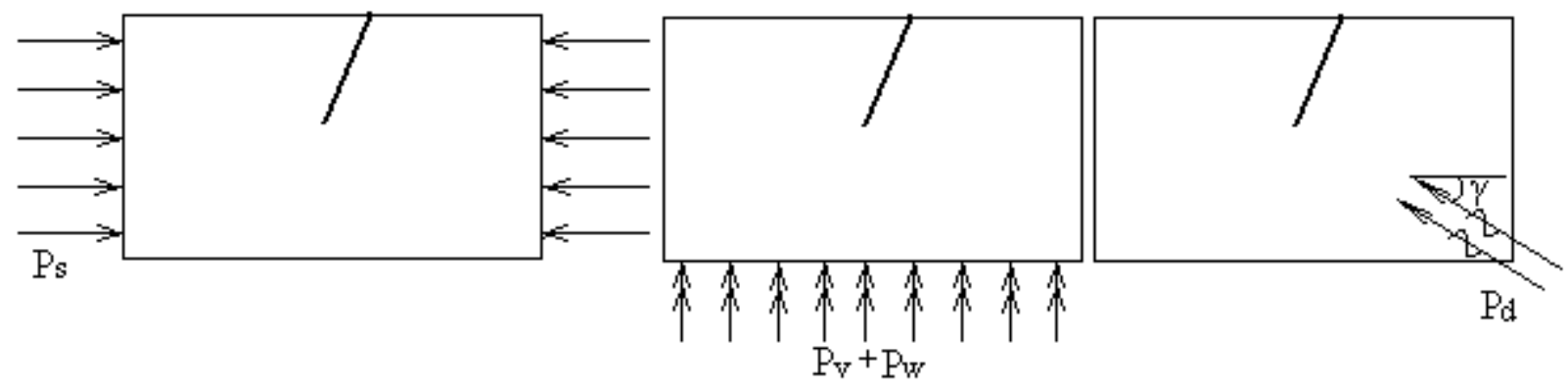

(a)under horizontal static load; (b)under vertical static load and water pressure; (c)under dynamic load

Fig.3 Loads decomposition 
In Fig.3(a), $\quad K_{\mathrm{I}}^{a}=P_{s} \sin ^{2} \alpha \sqrt{\pi a}$

$$
K_{\mathrm{II}}{ }^{a}=P_{s} \sin \alpha \cos \alpha \sqrt{\pi a}
$$

In Fig.3(b), due to the inclined fault stress intensity factor hard to find, it is simplified into a vertical fault ${ }^{[9]}$, then

$K_{\mathrm{I}}^{b}=\left(P_{v}+P_{w}\right) l f\left(\frac{a}{W}\right) / W^{3 / 2}$

In Eq.3, $f\left(\frac{a}{W}\right)=2.9\left(\frac{a}{W}\right)^{1 / 2}-4.6\left(\frac{a}{W}\right)^{3 / 2}+21.8\left(\frac{a}{W}\right)^{5 / 2}-37.6\left(\frac{a}{W}\right)^{7 / 2}+38.7\left(\frac{a}{W}\right)^{9 / 2}$

In Fig.3(c), $K_{\mathrm{I}}(t)=P_{d} \sin (\alpha+\gamma) \sqrt{c_{2} t} \frac{2 \sqrt{2}}{\sqrt{\pi}} \kappa \frac{c_{R}}{c_{2}}$

$$
K_{\mathrm{II}}(t)=P_{d} \cos (\alpha+\gamma) \sqrt{c_{2} t} \frac{2 \sqrt{2}}{\sqrt{\pi}} \kappa \frac{c_{R}}{c_{2}}
$$

In Eq.4 and Eq.5, $\kappa=\sqrt{c_{2} / c_{1}} ;$ Longitudinal wave velocity: $c_{1}=\sqrt{\frac{E(1-v)}{\rho(1+v)(1-2 v)}}$; Shear wave velocity: $\quad c_{2}=\sqrt{\frac{E}{2 \rho(1+v)}} ; \quad c_{R}$ is the Rayleigh wave velocity,drawn from the minimum real root of following equation.

$$
\left(2-\frac{c_{R}{ }^{2}}{c_{2}{ }^{2}}\right)^{2}-4\left(1-\frac{c_{2}{ }^{2}}{c_{1}{ }^{2}} \frac{c_{R}{ }^{2}}{c_{2}{ }^{2}}\right)^{1 / 2}\left(2-\frac{c_{R}{ }^{2}}{{c_{2}}^{2}}\right)^{1 / 2}=0 ;
$$

$E$ is the Modulus of elasticity; $v$ is the Poisson's ratio. Therefore, the fault tip Stress Intensity Factors can be concluded.

$$
\begin{aligned}
& K_{\mathrm{I}}=P_{s} \sin ^{2} \alpha \sqrt{\pi a}+\left(P_{v}+P_{w}\right) l f\left(\frac{a}{w}\right) / W^{3 / 2}+P_{d} \sin (\alpha+\gamma) \sqrt{c_{2} t} \frac{2 \sqrt{2}}{\sqrt{\pi}} \kappa \frac{c_{R}}{c_{2}} \\
& K_{\mathrm{II}}=P_{s} \sin \alpha \cos \alpha \sqrt{\pi a}+P_{d} \cos (\alpha+\gamma) \sqrt{c_{2} t} \frac{2 \sqrt{2}}{\sqrt{\pi}} \kappa \frac{c_{R}}{c_{2}}
\end{aligned}
$$

For I-II composite crack, Strain Energy Density Factor Criterion can be used to judge whether the crack initial. The Strain energy density factor is as Eq.8

$$
\begin{aligned}
& S(\theta)=a_{11} K_{\mathrm{I}}{ }^{2}+a_{12} K_{\mathrm{I}} K_{\mathrm{II}}+a_{22} K_{\mathrm{II}}{ }^{2} \\
& \text { In Eq.8, } a_{11}=\frac{1}{16 \pi G}\left[k+(k-1) \cos \theta-\cos ^{2} \theta\right] \text {, } \\
& a_{12}=\frac{1}{16 \pi G}[-(k+1) \cos \theta+2 \sin \theta \cos \theta] \\
& a_{22}=\frac{1}{16 \pi G}\left[k-(k-1) \cos \theta+3 \cos ^{2} \theta\right]
\end{aligned}
$$

$\mathrm{G}$ is the material's shear modulus. For plain strain, $k=3-4 v, v$ is the material's Poisson ratio.

In the same external conditions, when loading the certain rate dynamic load, the strain energy density factor of I、 II type compound crack in the same materials is Constant $S_{c r}$. That is to say, in the above conditions, when the strain energy density factor of composite crack tip reaches a certain value $S_{c r}$, the cracks are beginning to initiate.

\section{Discuss}

Seen from Eq.6 to Eq.8, flack or crack's activation is related with water pressure, vertical pressure, horizontal pressure, flack's thickness and dip angle, dynamic load, dynamic load's direction and duration time and so on. 
If the following conditions can meet

$$
P_{v}=\rho_{r} g h, P_{s}=\frac{v}{1-2 v} P_{v}=\frac{v}{1-2 v} \rho_{r} g h, P_{w}=\rho_{w} g h
$$

The fault tip Stress Intensity Factors is as follows:

$$
\begin{aligned}
& K_{\mathrm{I}}=\frac{v}{1-2 v} \rho_{r} g h \sin ^{2} \alpha \sqrt{\pi a}+\left(\rho_{v}+\rho_{w}\right) \cdot g h l f\left(\frac{a}{W}\right) / W^{3 / 2}+P_{d} \sin (\alpha+\gamma) \sqrt{c_{2} t} \frac{2 \sqrt{2}}{\sqrt{\pi}} \kappa \frac{c_{R}}{c_{2}} \\
& K_{\mathrm{II}}=\frac{v}{1-2 v} \rho_{r} g h \sin \alpha \cos \alpha \sqrt{\pi a}+P_{d} \cos (\alpha+\gamma) \sqrt{c_{2} t} \frac{2 \sqrt{2}}{\sqrt{\pi}} \kappa \frac{c_{R}}{c_{2}}
\end{aligned}
$$

Analysis Eq.8 -Eq.10, the strain energy density factor increases with the depth of rock mass. In deep depth, when depth " $h$ " is large enough, the strain energy density factor is very close to the initial strain energy density factor " $S_{c r}$ ". At this time, a very small load disturbances may make the strain energy density factor reach " $S_{c r}$ ” values, resulting in fault or crack initiation.

Strain energy density factor have great relations with fault parameters: it decreases with fault thickness "W" increase, increases with the fault deep "a” growth. Strain energy density factor has positive growth relationship with the dynamic load and time, i.e. $S(\theta) \propto P_{d} \sqrt{t}$. If the underground stope exists blasting and other dynamic load, when there is a fault in the floor, not only the blasting mining intensity, but also the duration time should be considered.

\section{Summaries}

1) Inadequacy of the current fault water inrush mechanism is analyzed, point out water inrush from faults not only relative with the static load but also with dynamic loads, especially in deep stope, rock mass in high stress field, small dynamic load may lead to fault activation.

2) Based on fracture mechanics, fault activation model is established and the stress intensity factor is calculated. For the deep fault, fault model takes into account the height, fault's depth, span, thickness, water pressure and other factors' influence.

3) Strain energy density factor has great relations with fault parameters: It decreases with fault thickness "W" increase, increases with the fault deep "a" and stope span growth.

4) Strain energy density factor has positive growth relationship with the dynamic load and time, $S(\theta) \propto P_{d} \sqrt{t}$. If the underground stope exists blasting and other dynamic load, when there is a fault in the floor, not only the blasting mining intensity but also the duration time should be considered.

\section{Acknowledgment}

Fund Project: Supported by the National Natural Science Foundation of China(No.51174228, No.310972238)

\section{References}

[1] Zhang Lihai, Zhang Yecheng. Characteristic and generate condition of coal mine water bursting calamity in China [J], China Mining Magazine, 2008, Vol. 17 (2):44-46

[2] Tan Zhixiang. Mechanism analysis of water inrush from fault[J]. Mining safety \& environmental protection, 1999(3):21-23.

[3] Yang Xin'an, Chen Jun, Yang Xizeng. Study on the water invasion classification and mechanism in Fengfeng mine area[J]. Journal of Geo logical Hazards and Environment Preservation, 1999,Vol.10(2):24-29

[4] WU Qiang, ZHOU Yingjie, LIU Jintao et al. Mechanical experiment study on lag mechanism of water-bursting of fault under coal seam [J]. JOURNAL OF CHINA COAL SOCIETY, 2003, Vol.28 (6):561-565

[5] Yin Huiyong, Wei Jiuchuan, Li Zhilin et al. Analysis on Water Inrush Mechanism in Fracture 
Tectonics of Panxi Coalmine [J]. Journal of Shandong University of Science and Technology, 2007, Vol.26 (1):30-34

[6] Zhang Jincai. Investigations of water inrushes from aquifers under coal seams[J]. International Journal of Rock Mechanics \& Mining Sciences, 2005, 42: 350-360

[7] Li Jianmin, Zhu Bin, Wu Qiang. Mechanical mechanism analysis of water inrush condition on mechanized top coal caving in steep seam in Zhaogezhuang coalmine [J]. Journal of China Coal Society, Vol.32 (5):453-457

[8] ZHU Qing-hua, FENG Mei-mei, MAO Xian-biao. Numerical analysis of water inrush from working-face floor during mining [J], Journal of China University of Mining \& Technology, 2008, Vol.18 (2):159-163

[9] LIU Baochen. Measuring technique of experimental fracture and damage mechanics[M]. Mechanical Technology Press, 1994:305. 